\title{
Rebuttal to Drs. Grinberg and Heinsen
}

\author{
Sarah K. Kaufman ${ }^{1} \cdot$ Kelly Del Tredici $^{2} \cdot$ Heiko Braak $^{2} \cdot$ Marc I. Diamond $^{1}$
}

Received: 28 September 2018 / Accepted: 28 September 2018 / Published online: 3 October 2018

c) Springer-Verlag GmbH Germany, part of Springer Nature 2018

Drs. Heinsen and Grinberg suggest that because of sampling bias and insufficient sensitivity of the tau seeding assay, our methods miss "hidden" seeding activity in the locus coeruleus (LC) at early stages of Alzheimer's disease (AD), and that our conclusion that the LC is unlikely to be the origin of pathological seeding in $\mathrm{AD}$ is incorrect. We strongly disagree, and would like to make several points.

1. With regard to sampling bias, punch biopsies were taken in a highly reproducible manner using serial sections through the lower brainstem to be sure of the anatomy. All cases evaluated the same essential brain region - the mid-LC. We included some of the neuropil immediately surrounding the LC to preserve the integrity of the LC because a biopsy punch with a smaller diameter might miss some LC neurons. AT8 signal (when so indicated) was clearly positive in the neurons of the sampled LC portion.

2. This work tested the hypothesis that the density of neurofibrillary tangle (NFT) pathology as detected by AT8 immunostaining has a strong correlation with seeding activity in AD. This is clearly not the case. Even if we exclude the LC from consideration, we easily observed seeding activity in cortical regions with very low levels of AT8-positive tau deposition (by definition, since these were cases from early/mid NFT stages). The assay is clearly sensitive enough to detect robust seeding activity even in the absence of prominent AT8 tau pathology.

3. The argument that the seeding assay is not sensitive enough to detect activity in the numerous AT8-positive neurons of the LC (even given an overall reduced density vs. the cortex) is not tenable. Signal intensity in "positive" cases was 10-100 $\times$ above the signal in "negative" ones. We refer skeptics to Fig. 1d in Kaufman et al., in which seeding activity from formalin-fixed brain tis-

\footnotetext{
Marc I. Diamond

marc.diamond@utsouthwestern.edu

UT Southwestern, Dallas, TX, USA

2 University of Ulm, Ulm, Germany
}

sue (comparable to what we detect in the LC) was easily scored even after 100-fold dilution [1]. It is highly unlikely that a marginal reduction (even several fold) in neuron density in the LC would cause us to miss seeding activity from the many AT8-positive LC neurons.

4. The LC can clearly produce seeding at the level observed in the trans/entorhinal cortices and other regions, regardless of nerve cell type or neuronal quantity. By NFT Stage III, seeding in the LC was as robust as that observed in other regions with higher neuronal density (and often with fewer AT8-positive neurons). Even ignoring the comparable levels of seeding activity that can be detected in these different regions, Figs. 4a and 5 in Kaufman et al. indicate virtually no brains were seed positive in the LC and negative in the TRE [2]. The opposite, however, is striking: the TRE reliably exhibited seeding positivity in many samples while the LC scored below threshold.

We wholeheartedly agree with Drs. Grinberg and Heinsen that further studies are needed. While single-cell analyses of seeding activity and normalization to total tau protein were not feasible in our study, we look forward to employing these methods in future work. We also agree that without a survey of many brain regions, independent of whether they typically show AT8-positive signal, we cannot precisely pinpoint the origin of seeding activity in AD or other tauopathies. At this point, we only reaffirm our principle conclusion that our data are not consistent with the LC as the origin of tau seeding activity in AD.

\section{References}

1. Kaufman SK, Thomas TL, Del Tredici K, Braak H, Diamond MI (2017) Characterization of tau prion seeding activity and strains from formaldehyde-fixed tissue. Acta Neuropathol Commun $5(1): 41$

2. Kaufman SK, Del Tredici K, Thomas TL, Braak H, Diamond MI (2018) Tau seeding activity begins in the transentorhinal/entorhinal regions and anticipates phospho-tau pathology in Alzheimer's disease and PART. Acta Neuropathol 136(1):57-67 